\title{
Reactivity of 3-Cyanoacetylindole Derivatives: Synthesis of 3-Hydrazonopyrazolyl and 3-Thiadiazolyl Indole Derivatives
}

\author{
Hamdi M. Hassaneen", Huwaida M. E. Hassaneen, Zakaria Ahmed Gomaa \\ Department of Chemistry, Faculty of Science, Cairo University, Giza, Egypt \\ E-mail:"huwaidahassaneen@hotmail.com \\ Received May 27, 2011; revised June 27, 2011; accepted July 5, 2011
}

\begin{abstract}
The coupling reaction of 3-cyanoacetyl-2-methylindole 1a with the aromatic diazonium salts gave the corresponding arylhydrazones 2a-e. Compounds 2 were used for synthesis of 4-aminopyrazole-5-carbonitrile 4a-e and 5-amino-4-arylazo-3-pyrazoles 5a-e derivatives. Also, treatment of 3-cyanoacetyl-2-phenylindole 1b with phenyl isothiocyanate gave the corresponding thioacetanilide 7. The later compound $\mathbf{7}$ was utilized as the key intermediate for the synthesis of some new thiadiazole derivatives 9a-r. The structures of all new compounds were elucidated on the basis of elemental analysis and spectral data.
\end{abstract}

Keywords: 3-Methyl Indole, 3-Phenyl Indole, Phenyl Isothiocyanate, Cyanoacetic Acid

\section{Introduction}

The indole moiety is found in various pharmacologically and biologically active compounds [1,2]. Many indole alkaloids are recognized as one of the rapidly growing groups of marine invertebrate metabolites for their broad spectrum of biological properties [3-6]. For example, five novel indole alkaloids $[7,8]$, tunicate aplidium meridianum A-E, have been isolated from tunical splidium meridianum. They show cytotoxicity toward murine tumor cell lines and have potent inhibition against several protein kinases $[9,10]$. Along with these, the substitution at the 3-position of the indole ring can take place by connecting an additional heterocyclic ring, such as imidazole (topsentins [11,12], nortopsentins [13]), dihydroimidazole (disc odermindole [14]), oxazole (martefragin [15], amazole [16]), oxadiazine (alboinon [17]), maleimide (didemidines [18]), and piperazine (dragmacidone [19]). Therefore, 3-substituted indoles still represent a significant synthetic challenge.

\section{Results and Discussion}

As a part of our program aimed at developing a synthesis for pyrazole [20] and thiadiazole derivatives [21-23], we report here an efficient synthesis for aminopyrazoles, which are used as precursors for biologically active fused pyrazoles. Thus, reacting 2-methylindole with cyanoacetic acid in acetic anhydride, utilizing a literature procedure [24], led to formation of 3-cyanoacetyl-2-methylindole 1a. The latter compound $\mathbf{1}$ reacted with aromatic diazonium salts to yield the corresponding arylhydrazones 2a-e in excellent yields (Scheme 1). The $E$-structure for hydrazones 2A was preferred over possible hydrogen-bonded $Z$-structure 2B based on analog of the recently-reported structure of 3-substituted-2-aryl-hydrazono-3-oxoalkanenitriles, whose E-structure has confirmed by X-ray crystal structure determination [25] and supported by theoretical calculation [26].

Compound $\mathbf{2}$ reacted readily with chloroacetonitrile in presence of triethylamine to give products of molecular

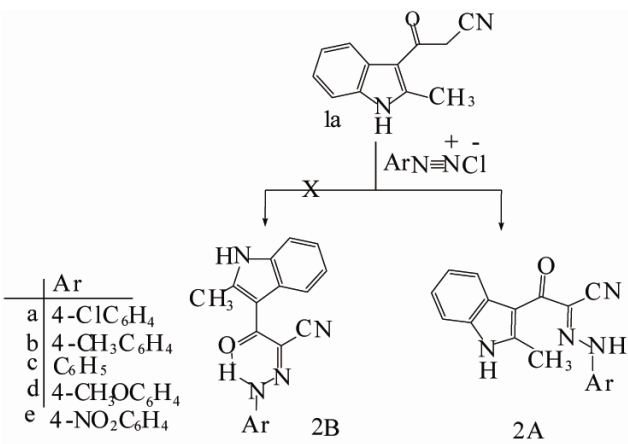

Scheme 1. MCR of $\alpha$-cyanoketenes S, S-acetals, amine and guanidine carbonate. 
formula corresponding to structure $\mathbf{4}$ or its isomeric structure $\mathbf{3}$ in excellent yield. Structure $\mathbf{4}$ was readily established based on ${ }^{1} \mathrm{H}$ NMR data which revealed the absence of a signal for the methylene group and appearance of two deuterium oxide exchangeable protons at $\delta 6.40$ ppm for amino group. it is believed that 4a-e has resulted from in stiu cyclization of the initially formed 3a-e (Scheme 2). Also, treatment of 2a-e with hydrazine hydrate in refluxing ethanol gave 5-amino-4-arylazo-3- (indole-3'-yl)pyrazoles 5a-e in excellent yield (Scheme 2). The structures of the products $5 \mathrm{a}-\mathrm{e}$ were established on the basis of elemental analysis and spectral data (see exeperimental).

It is known that a great variety of reactants bearing the $\mathrm{N}=\mathrm{C}=\mathrm{S}$ fragment undergo cyclization on reaction of hydrazonoyl halide compounds to afford thiadiazole derivatives [21-23], which have been shown to exhibit antiprotozoal [27] and fungicidal properties [28]. Thus, the base-catalyzed reaction of the 3-cyanoacetyl-2-phenylindole 1b with phenyl isothiocyanate in dry DMF at room temperature yielded the non-isolable potassium salt which by treatment with dilute hydrochloric acid gave the corresponding thioacetanilide 7 (Scheme 3). The structure of 7 was confirmed based on analytical and spectral data (see experimental section). For example $7 \mathrm{had}$ characteristic absorption peaks in its IR spectrum at 3343, $2202,1675 \mathrm{~cm}^{-1}$ due to $\mathrm{NH}, \mathrm{CN}, \mathrm{CO}$ groups respectively. In addition, the mass spectrum revealed a peak at $\mathrm{m} / \mathrm{z}=$ 395 corresponding to the molecular ion.

Treatment of thioacetanilide 7 with hydrazonoyl halides 8a-e in refluxing ethanol and in presence of triethylamine, afforded, in each case, only the 1,3,4-thiadiazoles 9a-e (Scheme 4). Elemental analyses and spectral data of the reaction products were in complete agreement with the proposed structures. For example 9b had characteristic absorption peaks in its IR spectrum at $3327,2195 \mathrm{~cm}^{-1}$ due to $\mathrm{NH}, \mathrm{CN}$ groups respectively. In addition, the mass spectrum revealed a peak at $\mathrm{m} / \mathrm{z}=522$ corresponding to its molecular ion (see experimental section).

To study the effect of carbonyl group of hydrazonoyl halide in the cyclization reaction, the thioacetanilide 7 was treated with hydrazonoyl halides 8f-r in refluxing ethanol and in presence of triethylamine, afforded, in each case, only one isomer 1,3,4-thiadiazole derivatives 9f-r (Scheme 5), which indicate that, there is no effect of the presence of carbonyl group in the cyclization reaction. Elemental analyses and spectral data of the reaction products were in complete agreement with the proposed structures. For example, 9k had characteristic absorption peaks in its IR spectrum at $3304,2195,1693 \mathrm{~cm}^{-1}$ due to $\mathrm{NH}, \mathrm{CN}, \mathrm{CO}$ groups respectively. In addition, the mass spectrum revealed a peak at $\mathrm{m} / \mathrm{z}=476$ corresponding to
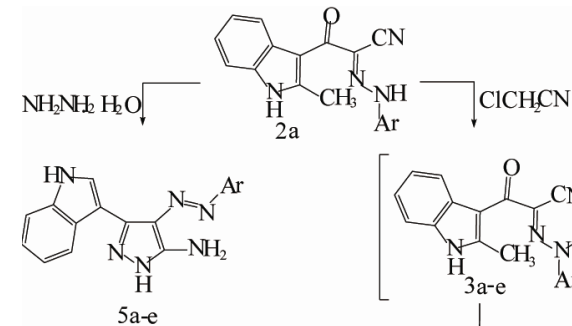

$$
\begin{array}{l|l} 
& \mathrm{Ar} \\
\hline \mathrm{a} & 4-\mathrm{ClC}_{6} \mathrm{H}_{4} \\
\mathrm{~b} & 4-\mathrm{CH}_{3} \mathrm{C}_{6} \mathrm{H}_{4} \\
\mathrm{c} & \mathrm{C}_{6} \mathrm{H}_{5} \\
\text { d } & 4-\mathrm{CH}_{3} \mathrm{OC}_{6} \mathrm{H}_{4} \\
\text { e } & 4-\mathrm{NO}_{2} \mathrm{C}_{6} \mathrm{H}_{4}
\end{array}
$$

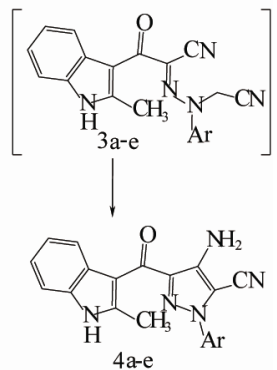

Scheme 2. Synthesis of amino-pyrazole derivatives 4 and 5.

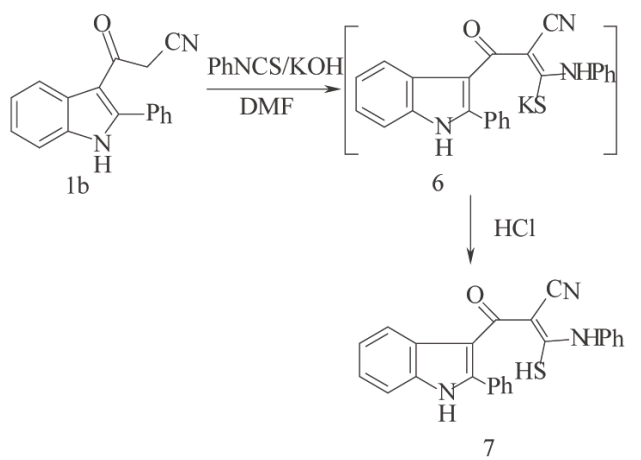

Scheme 3. Synthesis of thioacetanilide 7.

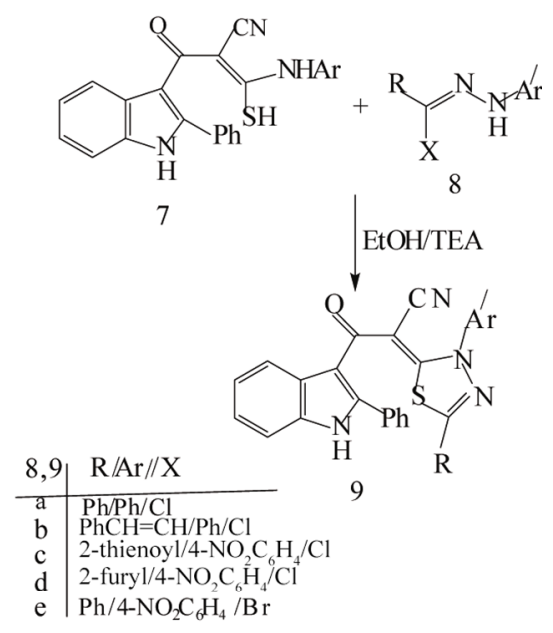

Scheme 4. 1,3,4-thiadiazoles 9.

the molecular ion. Its ${ }^{1} \mathrm{H}$ NMR showed two singlet signals at $\delta=2.35$ and $2.61 \mathrm{ppm}$ due to two methyl groups, in addition to one singlet signal due to proton at $\delta 11.9$ ppm represents $\mathrm{NH}$ group. Also, the ${ }^{13} \mathrm{C}$ NMR spectrum displayed characteristic signals at $\delta=190.40,184.27$, $114.7,25.91,20.79 \mathrm{ppm}$ due to $2 \mathrm{CO}, \mathrm{CN}, 2 \mathrm{CH}_{3}$ carbons respectively, in addition to all the other carbons at the expected chemical shifts (Scheme 5). 


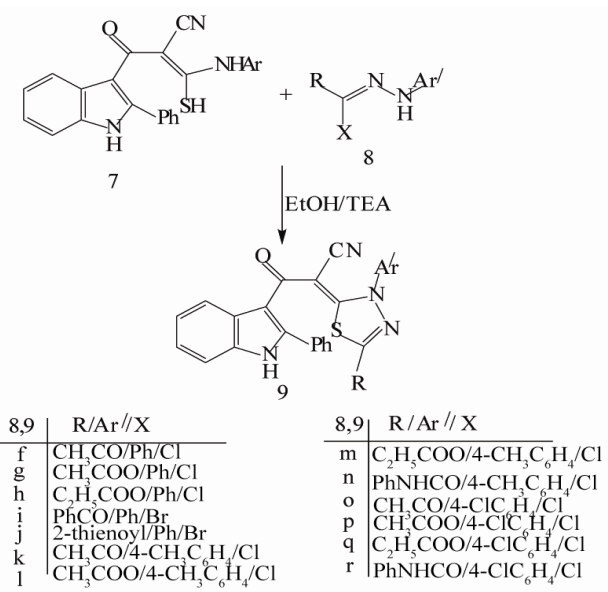

Scheme 5. Synthesis of 1,3,4-thiadiazoles 9 .

\section{Experimental Section}

\subsection{General}

All melting points were determined on an electrothermal GallenKamp melting point apparatus and are uncorrected. The IR spectra were recorded as KBr Pellets on a Jasco FTIR-460 plus Fourier transform infrared spectrophotometer. ${ }^{1} \mathrm{H}$ and ${ }^{13} \mathrm{C}$ NMR spectra were recorded at (300 $\mathrm{MHz})$ and $(75 \mathrm{MHz})$ respectively on Varian EM-300 $\mathrm{MHz}$ spectrometer. Chemical shifts $(\delta)$ are given from TMS (ppm) as internal standard for ${ }^{1} \mathrm{H}$ NMR and ${ }^{13} \mathrm{C}$ NMR. Mass spectra were recorded on AEI MS 30 mass spectrometer operating at $70 \mathrm{eV}$. The elemental analyses were performed at the Microanalytical Center of Cairo University.

\subsection{General Method for Preparation of (E)-N'-aryl-2-(2-methyl-1H-indol-3-yl) -2-oxo-acetohydrazonoyl cyanide 2a-e}

A cold solution of aryldiazonium salt $(10 \mathrm{mmol})$ was prepared by adding a solution of sodium nitrite $(10 \mathrm{mmol}$ in water) to a cold solution of the aromatic amine hydrochloride $(10 \mathrm{mmol})$ with stirring. The resulting solution of the diazonium salt was added to a cold solution of compound 1a $(10 \mathrm{mmol})$ in pyridine $(100 \mathrm{~mL})$. The reaction mixture was stirred at room temperature for $30 \mathrm{~min}$. the solid product so formed was collected, washed with water, and crystallized from suitable solvent to afford 2a-e.

(E)-N'-(4-Chlorophenyl)-2-(2-methyl-1H-indol-3-yl)-

2-oxoacetohydrazonoyl cyanide $2 a$ :

Orange crystals; m.p: $244^{\circ} \mathrm{C}$ (acetonitrile); yield (85\%); IR $(\mathrm{KBr}): v=3299(\mathrm{NH}), 2204(\mathrm{CN}) \mathrm{cm}^{-1} ;{ }^{1} \mathrm{H}$ NMR $\left(\mathrm{DMSO}-d_{6}\right): \delta=2.50\left(\mathrm{~s}, 3 \mathrm{H}\right.$, indole- $\left.\mathrm{CH}_{3}\right), 7.01-7.72(\mathrm{~m}$, $8 \mathrm{H}, \mathrm{ArH}), 11.91(\mathrm{~s}, 1 \mathrm{H}, \mathrm{NH}), 12.02(\mathrm{~s}, 1 \mathrm{H}, \mathrm{NH}) . \mathrm{MS}$ : $\mathrm{m} / \mathrm{z}(\%)=336\left[\mathrm{M}^{+}\right], 307,210,158,130$. Anal. for $\mathrm{C}_{18} \mathrm{H}_{13} \mathrm{ClN}_{4} \mathrm{O}$ : calcd. C, 64.19; H, 3.89; Cl, 10.53; N, 16.64. found $\mathrm{C}, 63.88 ; \mathrm{H}, 3.56 ; \mathrm{Cl}, 10.20 ; \mathrm{N}, 16.31$.

(E)-2-(2-Methyl-1H-indol-3-yl)-2-oxo- $N$ '-p-tolylacetohydrazonoyl cyanide $2 b$ :

Yellow crystals; m.p: $207^{\circ} \mathrm{C}$ (methanol); yield ( $87 \%$ ); IR(KBr): $v=3348(\mathrm{NH}), 2216(\mathrm{CN}) \mathrm{cm}^{-1} ;{ }^{1} \mathrm{H}$ NMR $\left(\mathrm{DMSO}-d_{6}\right): \delta=2.21\left(\mathrm{~s}, 3 \mathrm{H}, \mathrm{CH}_{3}\right), 2.50(\mathrm{~s}, 3 \mathrm{H}$, indole- $\left.\mathrm{CH}_{3}\right), 7.04-7.75(\mathrm{~m}, 8 \mathrm{H}, \mathrm{Ar} \mathrm{H}), 11.85(\mathrm{~s}, 1 \mathrm{H}, \mathrm{NH})$, $11.98(\mathrm{~s}, 1 \mathrm{H}, \mathrm{NH}) .{ }^{13} \mathrm{C}$ NMR (DMSO- $\left.d_{6}\right): \delta=23.92$, $29.81,120.05,120.12,123.0,194.94,125.01,130.01$, $130.42,131.01,136.97,139.15,143.0,144.32,149.22$, 153.32, 192.56. MS: $\mathrm{m} / \mathrm{z}(\%)=316\left[\mathrm{M}^{+}\right], 287,210,158$, 130, 91. Anal. for $\mathrm{C}_{19} \mathrm{H}_{16} \mathrm{~N}_{4} \mathrm{O}$ : Calcd. C, 72.13; H, 5.1; N, 17.71. found $\mathrm{C}, 71.81 ; \mathrm{H}, 4.7 ; \mathrm{N}, 17.39$.

(E)-2-(2-Methyl-1H-indol-3-yl)-2-oxo- $N$ '-phenyl-Acetohydrazonoyl cyanide $2 c$ :

Yellow crystals ; m.p: $235^{\circ} \mathrm{C}$ (ethanol); yield $(83 \%)$; IR (KBr): $v=3366(\mathrm{NH}), 2200(\mathrm{CN}) \mathrm{cm}^{-1} ;{ }^{1} \mathrm{H}$ NMR $\left(\mathrm{DMSO}_{\mathrm{d}}\right): \delta=2.50\left(\mathrm{~s}, 3 \mathrm{H}\right.$, indole- $\left.\mathrm{CH}_{3}\right), 7.03-7.71(\mathrm{~m}$, 9H, ArH), 11.94 (s, 1H, NH), 11.98 (s, 1H, NH).MS: m/z $(\%)=302\left[\mathrm{M}^{+}\right], 273,210,158,130,77$. Anal. for $\mathrm{C}_{18} \mathrm{H}_{14} \mathrm{~N}_{4} \mathrm{O}$ : calcd. C, 71.51; H, 4.67; N, 18.53. found $\mathrm{C}$, 71.12; H, 4.41; N, 18.28 .

(E)-N'-(4-Methoxyphenyl)-2-(2-methyl-1H-indol-3-yl)2-oxoacetohydrazonoyl cyanide $2 d$ :

Yellow crystals; m.p: $194^{\circ} \mathrm{C}$ (ethanol); yield (77\%); IR $(\mathrm{KBr}): v=3265(\mathrm{NH}), 2201(\mathrm{CN}) \mathrm{cm}^{-1} ;{ }^{1} \mathrm{H}$ NMR $\left(\mathrm{DMSO}_{6}\right): \delta=2.50\left(\mathrm{~s}, 3 \mathrm{H}\right.$, indole- $\left.\mathrm{CH}_{3}\right), 3.69(\mathrm{~s}, 3 \mathrm{H}$, $\left.\mathrm{OCH}_{3}\right), 6.82$ - 7.69 (m, 8H, Ar H), 11.89 (s, 1H, NH), 11.98 (s, 1H, NH). MS: m/z (\%) = $332\left[\mathrm{M}^{+}\right], 158,130$. Anal. for $\mathrm{C}_{19} \mathrm{H}_{16} \mathrm{~N}_{4} \mathrm{O}_{2}$ : calcd. C, 68.66; $\mathrm{H}, 4.85 ; \mathrm{N}, 16.86$. found $\mathrm{C}, 68.29 ; \mathrm{H}, 4.46 ; \mathrm{N}, 16.38$.

(E)-2-(2-Methyl-1H-indol-3-yl)-N'-(4-nitrophenyl)-2oxoacetohydrazonoyl cyanide $2 e$ :

Orange crystals; m.p: $274^{\circ} \mathrm{C}$ (acetonitrile); yield (88\%); IR (KBr): $v=3266(\mathrm{NH}), 2218(\mathrm{CN}) \mathrm{cm}^{-1} ;{ }^{1} \mathrm{H}$ NMR $\left(\right.$ DMSO- $\left.d_{6}\right): \delta=2.52\left(\mathrm{~s}, 3 \mathrm{H}\right.$, indole- $\left.\mathrm{CH}_{3}\right), 7.02-8.16(\mathrm{~m}$, $8 \mathrm{H}, \mathrm{ArH}), 12.06$ (s, 1H, NH), 12.39 (s, 1H, NH).MS: m/z $(\%)=347\left[\mathrm{M}^{+}\right], 318,158,130$. Anal. for $\mathrm{C}_{18} \mathrm{H}_{13} \mathrm{~N}_{5} \mathrm{O}_{3}$ : calcd. C, 62.24; H, 3.77; N, 20.16. found C, 61.90; H, $3.46 ; \mathrm{N}, 19.89$.

\subsection{General Method for Preparation of 4-amino-1-aryl-3-(2-methyl-1H-indole-3-car bonyl)-1H-pyrazole-5-carbonitrile Derivatives 4a-e}

To a solution of $2(5 \mathrm{mmol})$ in triethylamine $(10 \mathrm{mmol})$, chloroacetonitrile $(16 \mathrm{mmol})$ was added. The reaction mixture was refluxed for $2 \mathrm{~h}$, and then poured onto cold dilute $\mathrm{HCl}$. The solid product formed was filtered off and crystallized from suitable solvent to afford 4a-e.

4-Amino-1-(4-chlorophenyl)-3-(2-methyl-1H-indole-3- 
carbonyl)-1H-pyrazole-5-carbonitrile $4 a$ :

Yellow crystals; m.p: $252^{\circ} \mathrm{C}$ (acetic acid); yield (88\%); IR $(\mathrm{KBr}): v=3471 \& 3353\left(\mathrm{NH}_{2}\right), 3313(\mathrm{NH}), 2212$ (CN) $\mathrm{cm}^{-1} ;{ }^{1} \mathrm{H}$ NMR (DMSO- $\left.d_{6}\right): \delta=2.50(\mathrm{~s}, 3 \mathrm{H}$, indole- $\left.\mathrm{CH}_{3}\right), 6.40$ (s, $\left.2 \mathrm{H}, \mathrm{NH}_{2}\right), 7.04-7.81(\mathrm{~m}, 8 \mathrm{H}, \mathrm{ArH})$, $12.02(\mathrm{~s}, 1 \mathrm{H}, \mathrm{NH}) . \mathrm{MS}: \mathrm{m} / \mathrm{z}(\%)=375\left[\mathrm{M}^{+}\right], 360,158$, 130. Anal. for $\mathrm{C}_{20} \mathrm{H}_{14} \mathrm{ClN}_{5} \mathrm{O}$ : calcd. $\mathrm{C}, 63.92 ; \mathrm{H}, 3.75 ; \mathrm{Cl}$, 9.43; N, 18.64. found $\mathrm{C}, 63.51 ; \mathrm{H}, 3.50 ; \mathrm{Cl}, 9.03$; $\mathrm{N}, 18.22$.

4-Amino-3-(2-methyl-1H-indole-3-carbonyl)-1-p-tolyl1H-pyrazole-5-carbonitrile $4 b$ :

Brown crystals; m.p: $188^{\circ} \mathrm{C}$ (methanol); yield (91\%); IR (KBr): $v=3473 \& 3356\left(\mathrm{NH}_{2}\right), 3312(\mathrm{NH}), 2212(\mathrm{CN})$ $\mathrm{cm}^{-1} ;{ }^{1} \mathrm{H}$ NMR (DMSO- $\left.d_{6}\right): \delta=2.21\left(\mathrm{~s}, 3 \mathrm{H}, \mathrm{CH}_{3}\right), 2.50$ $\left(\mathrm{s}, 3 \mathrm{H}\right.$, indole- $\left.\mathrm{CH}_{3}\right), 6.41\left(\mathrm{~s}, 2 \mathrm{H}, \mathrm{NH}_{2}\right), 7.05-7.79(\mathrm{~m}$, $8 \mathrm{H}, \mathrm{ArH}), 11.98(\mathrm{~s}, 1 \mathrm{H}, \mathrm{NH}) . \mathrm{MS}: \mathrm{m} / \mathrm{z}(\%)=355\left[\mathrm{M}^{+}\right]$, $340,225,158,130,91$. Anal. for $\mathrm{C}_{21} \mathrm{H}_{17} \mathrm{~N}_{5} \mathrm{O}$ : calcd. $\mathrm{C}$, 70.97; H, 4.82; N, 19.71. found $\mathrm{C}, 70.55 ; \mathrm{H}, 4.61 ; \mathrm{N}$, 19.33 .

4-Amino-3-(2-methyl-1H-indole-3-carbonyl)-1-phenyl1H-pyrazole-5-carbonitrile $4 c$ :

Brown crystals; m.p: $204^{\circ} \mathrm{C}$ (ethanol); yield (87\%); IR $(\mathrm{KBr}): v=3470 \& 3358\left(\mathrm{NH}_{2}\right), 3311(\mathrm{NH}), 2213(\mathrm{CN})$ $\mathrm{cm}^{-1} ;{ }^{1} \mathrm{H}$ NMR (DMSO- $\left.d_{6}\right): \delta=2.50\left(\mathrm{~s}, 3 \mathrm{H}\right.$, indole- $\left.\mathrm{CH}_{3}\right)$, $6.42\left(\mathrm{~s}, 2 \mathrm{H}, \mathrm{NH}_{2}\right), 7.03-7.81(\mathrm{~m}, 9 \mathrm{H}, \mathrm{ArH}), 12.0(\mathrm{~s}, 1 \mathrm{H}$, $\mathrm{NH})$. MS: $\mathrm{m} / \mathrm{z}(\%)=341\left[\mathrm{M}^{+}\right], 326,158,130,77$. Anal. for $\mathrm{C}_{20} \mathrm{H}_{15} \mathrm{~N}_{5} \mathrm{O}$ : calcd. C, 70.37; H, 4.43; N, 20.52. found C, 69.98; H, 4.11; N, 20.13.

4-Amino-1-(4-methoxyphenyl)-3-(2-methyl-1H-indole3-carbonyl)-1H-pyrazole-5-carbonitrile $4 d$ :

Brown crystals; m.p: $214^{\circ} \mathrm{C}$ (acetonitrile); yield (89\%); IR (KBr): $v=3471 \& 3358\left(\mathrm{NH}_{2}\right), 3313(\mathrm{NH}), 2213(\mathrm{CN})$ $\mathrm{cm}^{-1} ;{ }^{1} \mathrm{H}$ NMR (DMSO- $\left.d_{6}\right): \delta=2.50\left(\mathrm{~s}, 3 \mathrm{H}\right.$, indole- $\left.\mathrm{CH}_{3}\right)$, $3.82\left(\mathrm{~s}, 3 \mathrm{H}, \mathrm{OCH}_{3}\right), 6.40\left(\mathrm{~s}, 2 \mathrm{H}, \mathrm{NH}_{2}\right), 7.10-7.83(\mathrm{~m}$, $8 \mathrm{H}, \mathrm{Ar} \mathrm{H}), 11.95(\mathrm{~s}, 1 \mathrm{H}, \mathrm{NH}) . \mathrm{MS}: \mathrm{m} / \mathrm{z}(\%)=371\left[\mathrm{M}^{+}\right]$, $356,158,130,77$. Anal. for $\mathrm{C}_{21} \mathrm{H}_{17} \mathrm{~N}_{5} \mathrm{O}_{2}$ : calcd. C, 67.91; $\mathrm{H}, 4.61 ; \mathrm{N}, 18.86$. found $\mathrm{C}, 67.50 ; \mathrm{H}, 4.33 ; \mathrm{N}, 18.43$.

4-Amino-3-(2-methyl-1H-indole-3-carbonyl)-1-(4nitrophenyl)-1H-pyrazole-5-carbonitrile $4 e$ :

Brown crystals; m.p: $289^{\circ} \mathrm{C}$ (acetonitrile); yield (88\%); IR (KBr): $v=3472 \& 3355\left(\mathrm{NH}_{2}\right), 3311(\mathrm{NH}), 2217(\mathrm{CN})$ $\mathrm{cm}^{-1} ;{ }^{1} \mathrm{H}$ NMR (DMSO- $\left.d_{6}\right): \delta=2.50\left(\mathrm{~s}, 3 \mathrm{H}\right.$, indole- $\left.\mathrm{CH}_{3}\right)$, $6.54\left(\mathrm{~s}, 2 \mathrm{H}, \mathrm{NH}_{2}\right), 7.08-8.46(\mathrm{~m}, 8 \mathrm{H}, \mathrm{ArH}), 12.06(\mathrm{~s}, 1 \mathrm{H}$, $\mathrm{NH})$. MS: $\mathrm{m} / \mathrm{z}(\%)=386\left[\mathrm{M}^{+}\right], 371,158,130,77$. Anal. for $\mathrm{C}_{20} \mathrm{H}_{14} \mathrm{~N}_{6} \mathrm{O}_{3}$ : calcd. C, 62.17; H, 3.65; N, 21.75 . found $\mathrm{C}, 61.80 ; \mathrm{H}, 3.41 ; \mathrm{N}, 21.33$.

\subsection{General Method for Preparation of (Z)-4-(2-Aryl-hydrazono)-5-(2-methyl-1H-in dol-3-yl)-4H-pyrazol-3-amine derivatives 5a-e}

To an appropriate compounds $2(10 \mathrm{mmol})$ hydrazine hydrate $(10 \mathrm{mmol})$ was added in ethanol $(20 \mathrm{~mL})$. The reaction mixture was refluxed for $6 \mathrm{~h}$, the solvent was evaporated and the crude product was collected then crystallized from benzene to afford the corresponding compounds 5a-e.

\section{(Z)-4-(2-(4-Chlorophenyl)hydrazono)-5-(2-methyl-}

1H-indol-3-yl)-4H-pyrazol-3-amine $5 a$ :

Orange crystals; m.p: $232^{\circ} \mathrm{C}$; yield $(87 \%)$; IR (KBr): $v$ $=3473 \& 3454\left(\mathrm{NH}_{2}\right), 3356(\mathrm{NH}) \mathrm{cm}^{-1} ;{ }^{1} \mathrm{H}$ NMR (DMSO- $\left.d_{6}\right): \delta=2.49$ (s, 3H, indole- $\left.\mathrm{CH}_{3}\right), 6.19(\mathrm{~s}, 2 \mathrm{H}$, $\left.\mathrm{NH}_{2}\right), 7.02-7.64(\mathrm{~m}, 8 \mathrm{H}, \mathrm{ArH}), 11.48(\mathrm{~s}, 1 \mathrm{H}, \mathrm{NH})$, $12.30(\mathrm{~s}, 1 \mathrm{H}, \mathrm{NH}) . \mathrm{MS}: \mathrm{m} / \mathrm{z}(\%)=350\left[\mathrm{M}^{+}\right], 335,224$. Anal.for $\mathrm{C}_{18} \mathrm{H}_{15} \mathrm{ClN}_{6}$ : calcd. C, 61.63; H, 4.31; $\mathrm{Cl}, 10.11$; $\mathrm{N}, 23.96$. found $\mathrm{C}, 61.29 ; \mathrm{H}, 4.10 ; \mathrm{Cl}, 9.75 ; \mathrm{N}, 23.48$.

(Z)-5-(2-Methyl-1H-indol-3-yl)-4-(2-p-tolylhydra-zono )-4H-pyrazol-3-amine $5 b$ :

Yellow crystals; m.p: $235^{\circ} \mathrm{C}$; yield $(89 \%)$; IR (KBr): $v$ $=3468 \& 3450\left(\mathrm{NH}_{2}\right), 3355(\mathrm{NH}) \mathrm{cm}^{-1} ;{ }^{1} \mathrm{H}$ NMR $\left(\mathrm{DMSO}_{-} d_{6}\right): \delta=2.29\left(\mathrm{~s}, 3 \mathrm{H}, \mathrm{CH}_{3}\right), 2.49(\mathrm{~s}, 3 \mathrm{H}$, indole- $\left.\mathrm{CH}_{3}\right), 6.15$ (s, $\left.2 \mathrm{H}, \mathrm{NH}_{2}\right), 7.01-7.53(\mathrm{~m}, 8 \mathrm{H}, \mathrm{ArH})$, $11.45(\mathrm{~s}, 1 \mathrm{H}, \mathrm{NH}), 12.28(\mathrm{~s}, 1 \mathrm{H}, \mathrm{NH}) . \mathrm{MS}: \mathrm{m} / \mathrm{z}(\%)=330$ $\left[\mathrm{M}^{+}\right], 315,224,91$. Anal. for $\mathrm{C}_{19} \mathrm{H}_{18} \mathrm{~N}_{6}$ : Calcd. C, 69.07; $\mathrm{H}, 5.49 ; \mathrm{N}, 25.44$. found $\mathrm{C}, 68.72 ; \mathrm{H}, 5.30 ; \mathrm{N}, 25.01$.

(Z)-5-(2-Methyl-1H-indol-3-yl)-4-(2-phenylhydrazono) -4H-pyrazol-3-amine 5c:

Yellow crystals; m.p: $202^{\circ} \mathrm{C}$; yield (85\%); IR (KBr): $v$ $=3468 \& 3450\left(\mathrm{NH}_{2}\right), 3353(\mathrm{NH}) \mathrm{cm}^{-1} ;{ }^{1} \mathrm{H}$ NMR $\left(\right.$ DMSO- $\left.d_{6}\right): \delta=2.50\left(\mathrm{~s}, 3 \mathrm{H}\right.$, indole- $\left.\mathrm{CH}_{3}\right), 6.21(\mathrm{~s}, 2 \mathrm{H}$, $\left.\mathrm{NH}_{2}\right), 7.0$ - $7.62(\mathrm{~m}, 9 \mathrm{H}, \mathrm{ArH}), 11.43(\mathrm{~s}, 1 \mathrm{H}, \mathrm{NH}), 12.28$ (s, $1 \mathrm{H}, \mathrm{NH}) . \mathrm{M}: \mathrm{m} / \mathrm{z}(\%)=316\left[\mathrm{M}^{+}\right], 301,224,77$. Anal. for $\mathrm{C}_{18} \mathrm{H}_{16} \mathrm{~N}_{6}$ : calcd. C, 68.34; H, 5.10; N, 26.56. found $\mathrm{C}$, 67.98; H, 4.82; N, 26.13.

(Z)-4-(2-(4-Methoxyphenyl)hydraono)-5-(2-methyl-

1H-indol-3-yl)-4H-pyrazol-3-amine $5 d$ :

Yellow crystals; m.p: $180^{\circ} \mathrm{C}$; yield $(80 \%) ; \mathrm{IR}(\mathrm{KBr}): v$ $=3389 \& 3187\left(\mathrm{NH}_{2}\right) \mathrm{cm}^{-1} ;{ }^{1} \mathrm{H}$ NMR (DMSO- $\left.d_{6}\right): \delta=$ $2.49\left(\mathrm{~s}, 3 \mathrm{H}\right.$, indole- $\left.\mathrm{CH}_{3}\right), 3.76\left(\mathrm{~s}, 3 \mathrm{H}, \mathrm{OCH}_{3}\right), 6.18(\mathrm{~s}, 2 \mathrm{H}$, $\left.\mathrm{NH}_{2}\right), 6.93-7.60(\mathrm{~m}, 8 \mathrm{H}, \mathrm{ArH}), 11.41(\mathrm{~s}, 1 \mathrm{H}, \mathrm{NH})$, 12.11 (s, $1 \mathrm{H}, \mathrm{NH}) . \mathrm{MS}: \mathrm{m} / \mathrm{z}(\%)=346\left[\mathrm{M}^{+}\right], 331,224$, 123. Anal. for $\mathrm{C}_{19} \mathrm{H}_{18} \mathrm{~N}_{6} \mathrm{O}$ : calcd. C, 65.88; H, 5.24; N, 24.26. found: $\mathrm{C}, 65.45 ; \mathrm{H}, 5.01 ; \mathrm{N}, 23.86$.

(Z)-5-(2-Methyl-1H-indol-3-yl)-4-(2-(4-nitrophenyl) hydrazono)-4H-pyrazol-3-amine 5 :

Red crystals; m.p: $285^{\circ} \mathrm{C}$; yield (81\%); IR (KBr): $v=$ $3467 \& 3399\left(\mathrm{NH}_{2}\right), 3349(\mathrm{NH}) \quad \mathrm{cm}^{-1} ;{ }^{1} \mathrm{H}$ NMR (DMSO- $\left.d_{6}\right): \delta=2.50\left(\mathrm{~s}, 3 \mathrm{H}\right.$, indole- $\left.\mathrm{CH}_{3}\right), 6.50(\mathrm{~s}, 2 \mathrm{H}$, $\left.\mathrm{NH}_{2}\right), 7.0-8.26(\mathrm{~m}, 8 \mathrm{H}, \mathrm{ArH}), 11.30(\mathrm{~s}, 1 \mathrm{H}, \mathrm{NH}), 12.18$ (s, $1 \mathrm{H}, \mathrm{NH}) . \mathrm{MS}: \mathrm{m} / \mathrm{z}(\%)=361\left[\mathrm{M}^{+}\right], 346,224$. Anal. for $\mathrm{C}_{18} \mathrm{H}_{15} \mathrm{~N}_{7} \mathrm{O}_{2}$ : calcd. C, 59.83; $\mathrm{H}, 4.18 ; \mathrm{N}, 27.13$. found $\mathrm{C}, 59.44 ; \mathrm{H}, 4.01 ; \mathrm{N}, 26.87$. 


\subsection{General Method for Preparation of (Z)-3mercapto-2-(2-phenyl-1H-indole-3-car bonyl)-3-(phenylamino) Acrylonitrile 7.}

To a stirred solution of potassium hydroxide $(0.56 \mathrm{~g}, 10$ $\mathrm{mmol})$ in dimethylformamide $(50 \mathrm{~mL})$, 3-cyan-oacetyl-2-phenylindole $\mathbf{1 b}(2.6 \mathrm{~g}, 10 \mathrm{mmol})$ was added. After stirring for $30 \mathrm{~min}$, phenyl isothiocyanate $(1.4 \mathrm{~g}, 10$ $\mathrm{mmol}$ ) was added, and stirring was continued for further $6 \mathrm{~h}$. The mixture was then poured over crushed ice containing hydrochloric acid. The solid product was filtered off, washed with water, dried and finally recrystallized from acetonitrile to afford 7.

(Z)-3-Mercapto-2-(2-phenyl-1H-indole-3-carbonyl)-3(phenylamino)acrylonitrile 7:

Yellow crystals; m.p. $186^{\circ} \mathrm{C}$; yield $(78 \%)$. IR (KBr): $v$ $=3343(\mathrm{NH}), 2202(\mathrm{CN}), 1676(\mathrm{CO}) \mathrm{cm}^{-1} . \mathrm{MS}: \mathrm{m} / \mathrm{z}(\%)$ $=395\left[\mathrm{M}^{+}\right]$. Anal. for $\mathrm{C}_{24} \mathrm{H}_{17} \mathrm{~N}_{3} \mathrm{OS}$ : calcd. $\mathrm{C}, 72.89 ; \mathrm{H}$, 4.33; N, 10.63; S, 8.11.found C, 72.45; H, 4.10; N, 10.47; S, 7.87 .

\subsection{General Procedure for the Preparation of 2-(3,5-Diaryl-1,3,4-thiadiazol-2-ylidene)-3-(2 -phenyl-1H-indole-3-yl)-3-oxo-propionitriles 9a-r .}

To a solution of the thioacetanilide $7(0.79 \mathrm{~g}, 2 \mathrm{mmol})$ in absolute ethanol $(20 \mathrm{~mL})$, hydrazonoyl halides 8 (2 $\mathrm{mmol})$ was added. To the resulting mixture triethylamine $(0.3 \mathrm{~mL})$ was added, and the reaction mixture was refluxed for $3 \mathrm{~h}$ and then cooled. The solid product was filtered off, washed with ethanol and crystallized from suitable solvent to afford the corresponding thiadiazole derivatives 9a-r.

(Z)-2-(3,5-diphenyl-1,3,4-thiadiazol-2(3H)-ylidene)-3oxo-3-(2-phenyl-1H-indol-3-yl)propane-nitrile $9 a$ :

Yellow crystals; m.p. $305^{\circ} \mathrm{C}$ (dioxane); yield (79\%). IR (KBr): $v=3277(\mathrm{NH}), 2199(\mathrm{CN}) \mathrm{cm}^{-1}$. MS: $\mathrm{m} / \mathrm{z} \mathrm{( \% )}$ $=496\left[\mathrm{M}^{+}\right]$. Anal. for $\mathrm{C}_{31} \mathrm{H}_{20} \mathrm{~N}_{4} \mathrm{OS}$ : calcd. C, 74.98; H, 4.06; N, 11.28; S, 6.46.found C, 74.49; H, 3.89; N, 10.92; $\mathrm{S}, 6.17$.

(Z)-3-Oxo-3-(2-phenyl-1H-indol-3-yl)-2-(3-phenyl-5-s tyryl-1,3,4-thiadiazol-2(3H)-ylidene)prop-anenitrile $9 b$ :

Yellow crystals; m.p. $299^{\circ} \mathrm{C}$ (acetonitrile); yield (73\%). IR (KBr): $v=3327(\mathrm{NH}), 2195(\mathrm{CN}) \mathrm{cm}^{-1} .{ }^{1} \mathrm{H}$ NMR $\left(\mathrm{CDCl}_{3}\right): \delta=7.18-7.9(\mathrm{~m}, 21 \mathrm{H}, \mathrm{ArH}), 8.8(\mathrm{~s}, 1 \mathrm{H}, \mathrm{NH})$. MS: $\mathrm{m} / \mathrm{z}(\%)=522\left[\mathrm{M}^{+}\right]$. Anal. for $\mathrm{C}_{33} \mathrm{H}_{22} \mathrm{~N}_{4} \mathrm{OS}$ : calcd. C, 75.84; H, 4.24; N, 10.72; S, 6.14.found C, 75.40; H, $4.01 ; \mathrm{N}, 10.43 ; \mathrm{S}, 5.86$.

(Z)-2-(3-(4-Nitrophenyl)-5-(thiophen-2-yl)-1,3,4-thiad ia-zol-2(3H)-ylidene)-3-oxo-3-(2-phenyl-1H-indol-3-yl) propanenitrile $9 c$ :
Yellow crystals; m.p. $361^{\circ} \mathrm{C}$ (dioxane); yield $(80 \%)$. IR (KBr): $v=3340(\mathrm{NH}), 2203(\mathrm{CN}) \mathrm{cm}^{-1}$. MS: $\mathrm{m} / \mathrm{z}(\%)$ $=547\left[\mathrm{M}^{+}\right]$. Anal. for $\mathrm{C}_{29} \mathrm{H}_{17} \mathrm{~N}_{5} \mathrm{O}_{3} \mathrm{~S}_{2}$ : calcd. $\mathrm{C}, 63.61 ; \mathrm{H}$, 3.13; N, 12.79 S, 11.71.found C, 63.19; H, 3.01; N, $12.45 ; \mathrm{S}, 11.35$.

(Z)-2-(5-(Furan-2-yl)-3-(4-nitrophenyl)-1,3,4-thiadiaz ol-2(3H)-ylidene)-3-oxo-3-(2-phenyl-1H-indol-3-yl) propanenitrile $9 d$ :

Yellow crystals; m.p. $328^{\circ} \mathrm{C}$ (acetonitrile); yield (78\%). IR (KBr): $v=3345(\mathrm{NH}), 2203(\mathrm{CN}) \mathrm{cm}^{-1}$. MS: $\mathrm{m} / \mathrm{z}(\%)$ $=531\left[\mathrm{M}^{+}\right]$.Anal. for $\mathrm{C}_{29} \mathrm{H}_{17} \mathrm{~N}_{5} \mathrm{O}_{4} \mathrm{~S}$ : calcd. $\mathrm{C}, 65.53 ; \mathrm{H}$, $3.22 ; \mathrm{N}, 13.18 ; \mathrm{S}, 6.03$. found: $\mathrm{C}, 65.11 ; \mathrm{H}, 3.0 ; \mathrm{N}, 12.84$; S, 5.73.

(Z)-2-(3-(4-Nitrophenyl)-5-phenyl-1,3,4-thiadiazol-2(3 H)-ylidene)-3-oxo-3-(2-phenyl-1H-indol-3-yl)propanenitrile $9 e$ :

Yellow crystals; m.p. $310^{\circ} \mathrm{C}$ (dioxane); yield (81\%). IR $(\mathrm{KBr}): v=3336(\mathrm{NH}), 2200(\mathrm{CN}) \mathrm{cm}^{-1}$. MS: $\mathrm{m} / \mathrm{z}(\%)=$ $541\left[\mathrm{M}^{+}\right]$. Anal. for $\mathrm{C}_{31} \mathrm{H}_{19} \mathrm{~N}_{5} \mathrm{O}_{3} \mathrm{~S}$ : calcd. C, 68.75; H, 3.54; N, 12.93; S, 5.92. found: C, 68.24; H, 3.31; N, 12.61; S, 5.70.

(Z)-2-(5-Acetyl-3-phenyl-1,3,4-thiadizol-2(3H)-ylidene) -3-oxo-3-(2-phenyl-1H-indol-3-yl)propanenitrile 9f:

Yellow crystals; m.p. $311^{\circ} \mathrm{C}$ (acetonitrile); yield (76\%). IR (KBr): $v=3300(\mathrm{NH}), 2194(\mathrm{CN}), 1693(\mathrm{CO}) \mathrm{cm}^{-1} \cdot{ }^{1} \mathrm{H}$ NMR $\left(\mathrm{CDCl}_{3}\right): \delta=2.67\left(\mathrm{~s}, 3 \mathrm{H}\right.$, indole- $\left.\mathrm{CH}_{3}\right), 7.19-8.21$ (m, 14H, Ar H), 8.56 (s, 1H, NH). MS: m/z (\%) = 462 $\left[\mathrm{M}^{+}\right]$. Anal. for $\mathrm{C}_{27} \mathrm{H}_{18} \mathrm{~N}_{4} \mathrm{O}_{2} \mathrm{~S}$ : calcd. C, 70.11; H, 3.92; N, 12.11; S, 6.93. found $\mathrm{C}, 69.51 ; \mathrm{H}, 3.73 ; \mathrm{N}, 11.81 ; \mathrm{S}$, 6.70 .

(Z)-Methyl-5-(1-cyano-2-oxo-2-(2-phenyl-1H-indol-3$y l)$ ethylidene)-4-phenyl-4,5-dihydro-1,3,4-thiadiazole-2carboxylate $9 g$ :

Yellow crystals; m.p. $247^{\circ} \mathrm{C}$ (acetonitrile); yield (73\%). IR (KBr): $v=3322(\mathrm{NH}), 2200(\mathrm{CN}), 1752(\mathrm{CO}), 1725$ (CO) $\mathrm{cm}^{-1} \cdot{ }^{1} \mathrm{H}$ NMR $\left(\mathrm{CDCl}_{3}\right): \delta=4.05$ (s, 3H, ester- $\left.\mathrm{CH}_{3}\right), 7.19-7.90(\mathrm{~m}, 14 \mathrm{H}, \mathrm{Ar} \mathrm{H}), 8.57(\mathrm{~s}, 1 \mathrm{H}, \mathrm{NH})$. MS: $\mathrm{m} / \mathrm{z}(\%)=478\left[\mathrm{M}^{+}\right]$. Anal. for $\mathrm{C}_{27} \mathrm{H}_{18} \mathrm{~N}_{4} \mathrm{O}_{3} \mathrm{~S}$ : calcd. C, 67.77; H, 3.79; N, 11.71; S, 6.70.found C, 67.40; H, $3.58 ; \mathrm{N}, 11.43 ; \mathrm{S}, 6.49$.

(Z)-Ethyl-5-(1-cyano-2-oxo-2-(2-phenyl-1H-indol-3-yl) ethylidene)-4-phenyl-4,5-dihydro-1,3,4-thiadiazole-2-carboxylate $9 h$ :

Yellow crystals; m.p. $272^{\circ} \mathrm{C}$ (acetonitrile); yield (74\%). IR (KBr): $v=3309(\mathrm{NH}), 2202(\mathrm{CN}), 1743(\mathrm{CO}), 1712$ (CO) $\mathrm{cm}^{-1} .{ }^{1} \mathrm{H}$ NMR (DMSO-d $\mathrm{d}_{6}$ ): $\delta=1.36$ (t, 3H, ester- $\left.\mathrm{CH}_{3}\right), 4.46\left(\mathrm{q}, 2 \mathrm{H}\right.$, ester- $\left.\mathrm{CH}_{2}\right), 7.09-7.63(\mathrm{~m}, 14 \mathrm{H}$, Ar H), 11.94 (s, 1H, NH). MS: m/z (\%) = $492\left[\mathrm{M}^{+}\right]$. Anal. for $\mathrm{C}_{28} \mathrm{H}_{20} \mathrm{~N}_{4} \mathrm{O}_{3} \mathrm{~S}$ : calcd. C, 68.28; H, 4.09; N, 11.37; S, 6.51.found C, 67.91; H, 3.92; N, 11.02; S, 6.25.

(Z)-2-(5-Benzoyl-3-phenyl-1,3,4-thiadiazol-2(3H)-ylid ene)-3-oxo-3-(2-phenyl-1H-indol-3-yl)-propanenitrile 9i:

Yellow crystals; m.p. $282^{\circ} \mathrm{C}$ (dioxane); yield (78\%). 
IR (KBr): $v=3316(\mathrm{NH}), 2202(\mathrm{CN}), 1650(\mathrm{CO}) \mathrm{cm}^{-1}$. MS: $\mathrm{m} / \mathrm{z}(\%)=524\left[\mathrm{M}^{+}\right]$. Anal. for $\mathrm{C}_{32} \mathrm{H}_{20} \mathrm{~N}_{4} \mathrm{O}_{2} \mathrm{~S}$ : calcd. C, 73.27; H, 3.84; N, 10.68; S, 6.11. found C, 72.92; H, $3.61 ; \mathrm{N}, 10.29 ; \mathrm{S}, 5.89$.

(Z)-3-Oxo-3-(2-phenyl-1H-indol-3-yl)-2-(3-phenyl-5(thiophene-2-carbonyl)-1,3,4-thiadiazol-2(3H)-ylidene) propanenitrile $9 j$ :

Yellow crystals; m.p. $303^{\circ} \mathrm{C}$ (acetonitrile); yield (72\%). IR (KBr): $v=3264(\mathrm{NH}), 2199(\mathrm{CN}), 1632(\mathrm{CO}) \mathrm{cm}^{-1}$. MS: $\mathrm{m} / \mathrm{z}(\%)=530\left[\mathrm{M}^{+}\right]$. Anal. for $\mathrm{C}_{30} \mathrm{H}_{18} \mathrm{~N}_{4} \mathrm{O}_{2} \mathrm{~S}_{2}$ : calcd. C, 67.91; H, 3.42; N, 10.56, S, 12.09. found C, 67.48; H, $3.21 ; \mathrm{N}, 10.28 ; \mathrm{S}, 11.81$.

(Z)-2-(5-Acetyl-3-p-tolyl-1,3,4-thiadiazol-2(3H)ylidene)-3-oxo-3-(2-phenyl-1H-indol-3-yl)propanenitrile $9 k$ :

Yellow crystals; m.p. $327^{\circ} \mathrm{C}$ (dioxane); yield (78\%). IR (KBr): $v=3304(\mathrm{NH}), 2195(\mathrm{CN}), 1693(\mathrm{CO}) \mathrm{cm}^{-1}$. ${ }^{1} \mathrm{H}$ NMR (DMSO-d $\mathrm{d}_{6}$ ): $\delta=2.35$ (s, 3H, $\left.\mathrm{CH}_{3}\right), 2.61(\mathrm{~s}, 3 \mathrm{H}$, $\left.\mathrm{CH}_{3}\right), 7.12-7.63(\mathrm{~m}, 13 \mathrm{H}, \mathrm{ArH}), 11.9(\mathrm{~s}, 1 \mathrm{H}, \mathrm{NH}){ }^{13} \mathrm{C}$ NMR (DMSO- $\left.d_{6}\right): \delta=20.79,25.91,66.26,79.28$, $111.45,114.70,119.68,120.51,122.32,126.58,127.17$, $128.31,128.51,129.38,131.80,135.55,139.90,140.25$, 155.68, 164.65, 184.27, 190.40. MS: $\mathrm{m} / \mathrm{z}(\%)=476\left[\mathrm{M}^{+}\right]$. Anal. for $\mathrm{C}_{28} \mathrm{H}_{20} \mathrm{~N}_{4} \mathrm{O}_{2} \mathrm{~S}$ : calcd. C, 70.57; H, 4.23; N, 11.76; S, 6.73.found C, 70.09; H, 4.02; N, 11.47; S, 6.49.

(Z)-Methyl5-(1-cyano-2-oxo-2-(2-phenyl-1H-indol-3-yl) ethylidene-4-p-tolyl-4,5-dihydro-1,3,4-thiadiazole-2-

carboxylate $9 l$ :

Yellow crystals; m.p. $270^{\circ} \mathrm{C}$ (dioxane); yield (82\%). IR (KBr): $v=3342(\mathrm{NH}), 2199(\mathrm{CN}), 1752(\mathrm{CO}), 1726$ (CO) $\mathrm{cm}^{-1}$. MS: $\mathrm{m} / \mathrm{z}(\%)=492\left[\mathrm{M}^{+}\right]$. Anal. for $\mathrm{C}_{28} \mathrm{H}_{20} \mathrm{~N}_{4} \mathrm{O}_{3} \mathrm{~S}$ : calcd. $\mathrm{C}, 68.28 ; \mathrm{H}, 4.24 ; \mathrm{N}, 11.37 ; \mathrm{S}$, 6.51.found C, 67.93; H, 4.01; N, 11.03; S, 6.28.

(Z)-Ethyl-5-(1-cyano-2-oxo-2-(2-phenyl-1H-indol-3-yl) ethylidene-4-p-tolyl-4,5-dihydro-1,3,4-thiadiazole-2carboxylate $9 \mathrm{~m}$ :

Yellow crystals; m.p. $280^{\circ} \mathrm{C}$ (acetonitrile); yield (73\%). IR (KBr): $v=3317(\mathrm{NH}), 2206(\mathrm{CN}), 1752(\mathrm{CO}), 1720$ (CO) $\mathrm{cm}^{-1} .{ }^{1} \mathrm{H}$ NMR (DMSO- $\left.d_{6}\right): \delta=1.35(\mathrm{t}, 3 \mathrm{H}$, ester- $\left.\mathrm{CH}_{3}\right), 2.34$ (s, 3H, $\left.\mathrm{CH}_{3}\right), 4.45$ (q, $2 \mathrm{H}$, ester- $\left.\mathrm{CH}_{2}\right), 7.09$ - $7.62(\mathrm{~m}, 13 \mathrm{H}, \mathrm{Ar} \mathrm{H}), 11.93$ (s, 1H, NH). MS: $\mathrm{m} / \mathrm{z}(\%)=$ $506\left[\mathrm{M}^{+}\right]$. Anal.for $\mathrm{C}_{29} \mathrm{H}_{22} \mathrm{~N}_{4} \mathrm{O}_{3} \mathrm{~S}$ : calcd. C, 68.76; H, 4.38; N, 11.06; S, 6.33.found C, 68.33; H, 4.15; N, 10.31; $\mathrm{S}, 6.14$.

(Z)-5-(1-Cyano-2-oxo-2-(2-phenyl-1H-indol-3-yl)ethyl idene)-N-phenyl-4-p-tolyl-4,5-dihydro-1,3,4-thiadiazole2-carboxamide $9 n$ :

Yellow crystals; m.p. $298^{\circ} \mathrm{C}$ (dioxane); yield (81\%). IR (KBr): $v=3276(\mathrm{NH}), 2207(\mathrm{CN}), 1667(\mathrm{CO}) \mathrm{cm}^{-1} .{ }^{1} \mathrm{H}$ NMR (DMSO- $\left.d_{6}\right): \delta=2.36\left(\mathrm{~s}, 3 \mathrm{H}, \mathrm{CH}_{3}\right), 7.24-7.82(\mathrm{~m}$, $18 \mathrm{H}, \mathrm{Ar} \mathrm{H}), 10.92(\mathrm{~s}, 1 \mathrm{H}, \mathrm{NH}), 11.87(\mathrm{~s}, 1 \mathrm{H}, \mathrm{NH}) .{ }^{13} \mathrm{C}$ NMR $\left(\right.$ DMSO- $\left.\mathrm{d}_{6}\right): \delta=20.79,66.24,78.92,111.53$, $114.91,119.72,120.41,120.65,120.93,122.23,124.73$,
$126.99,127.19,128.19,128.49,128.60,129.23,131.82$, $135.53,137.28,139.63,140.24,153.82,155.96,164.63$, 184.18. MS: $\mathrm{m} / \mathrm{z}(\%)=553\left[\mathrm{M}^{+}\right]$. Anal. for $\mathrm{C}_{33} \mathrm{H}_{23} \mathrm{~N}_{5} \mathrm{O}_{2} \mathrm{~S}$ : calcd. C, 71.59; H, 4.19; N, 12.65; S, 5.79.found $\mathrm{C}$, $71.12 ; \mathrm{H}, 3.98 ; \mathrm{N}, 12.30 ; \mathrm{S}, 5.61$.

(Z)-2-(5-Acetyl-3-(4-chlorophenyl)-1,3,4-thiadiazol-2(3H )-ylidene)-3-oxo-3-(2-phenyl-1H-indol-3-yl)propanenitrile 90 :

Yellow crystals; m.p. $347^{\circ} \mathrm{C}$ (dioxane); yield (81\%). IR (KBr): $v=3312(\mathrm{NH}), 2194(\mathrm{CN}), 1692(\mathrm{CO}) \mathrm{cm}^{-1}$. MS: $\mathrm{m} / \mathrm{z}(\%)=496\left[\mathrm{M}^{+}\right]$. Anal. for $\mathrm{C}_{27} \mathrm{H}_{17} \mathrm{ClN}_{4} \mathrm{O}_{2} \mathrm{~S}$ : calcd. C, 65.25; H, 3.45; Cl, 7.13; N, 11.27; S, 6.45. found $\mathrm{C}, 64.76 ; \mathrm{H}, 3.22 ; \mathrm{Cl}, 6.85 ; \mathrm{N}, 10.88 ; \mathrm{S}, 6.21$.

(Z)-Methyl-4-(4-chlorophenyl)-5-(1-cyano-2-(2-oxo-2(2-phenyl-1H-indol-3-yl)ethylidene)-4,5-dihydro-1,3,4thiadiazole-2-carboxylat $9 p$ :

Yellow crystals; m.p. $260^{\circ} \mathrm{C}$ (dioxane); yield (80\%). IR $(\mathrm{KBr}): v=3350(\mathrm{NH}), 2197(\mathrm{CN}), 1757(\mathrm{CO}) \mathrm{cm}^{-1} \cdot{ }^{1} \mathrm{H}$ NMR $\left(\right.$ DMSO- $\left.d_{6}\right): \delta=3.98\left(\mathrm{~s}, 3 \mathrm{H}\right.$, ester- $\left.\mathrm{CH}_{3}\right), 7.11-$ $7.68\left(\mathrm{~m}, 13 \mathrm{H}\right.$, Ar H) $11.99(\mathrm{~s}, 1 \mathrm{H}, \mathrm{NH}) \cdot{ }^{13} \mathrm{C}$ NMR $\left(\right.$ DMSO-d $\left._{6}\right): \delta=53.84,66.32,79.15,111.14,111.70$, $114.91,119.82,120.72,122.49,127.25,128.43,128.49$, 128.67, 128.87, 129.09, 131.85, 135.14, 135.63, 136.82, 140.37, 149.24, 158.33, 164.59, 183.98. MS: $\mathrm{m} / \mathrm{z}(\%)=$ $512\left[\mathrm{M}^{+}\right]$. Anal.for $\mathrm{C}_{27} \mathrm{H}_{17} \mathrm{ClN}_{4} \mathrm{O}_{3} \mathrm{~S}$ : calcd. C, 63.22; H, 3.34; Cl, 6.91; N, 10.92; S, 6.25.found: C, 62.81; H, 3.20; $\mathrm{Cl}, 6.65 ; \mathrm{N}, 10.59 ; \mathrm{S}, 6.01$.

(Z)-Ethyl4-(4-chlorophenyl)-5-(1-cyano-2-(2-oxo-2-(2phenyl-1H-indol-3-yl)ethylidene)-4,5-dihydro-1,3,4thiadiazole-2-carboxylate $9 q$ :

Yellow crystals; m.p. $266^{\circ} \mathrm{C}$ (acetonitrile); yield (79\%). IR (KBr): $v=3302(\mathrm{NH}), 2202(\mathrm{CN}), 1758(\mathrm{CO}), 1720$ (CO) $\mathrm{cm}^{-1}$. MS: $\mathrm{m} / \mathrm{z}(\%)=526\left[\mathrm{M}^{+}\right]$. Anal. for $\mathrm{C}_{28} \mathrm{H}_{19} \mathrm{ClN}_{4} \mathrm{O}_{3} \mathrm{~S}$ : calcd. C, 63.81; H, 3.63; Cl, 6.73; N, 10.63; S, 6.08.found $\mathrm{C}, 63.39 ; \mathrm{H}, 3.37 ; \mathrm{Cl}, 6.45 ; \mathrm{N}$, $10.30 ; \mathrm{S}, 5.81$.

(Z)-4-(4-Chlorophenyl)-5-(1-cyano-2-(2-oxo-2-(2phenyl-1H-indol-3-yl)ethylidene)-N-phenyl-4,5-dihydro1,3,4-thiadiazole-2-carboxamide $9 r$ :

Yellow crystals; m.p. $328^{\circ} \mathrm{C}$ (dioxane); yield (83\%). IR (KBr): $v=3289(\mathrm{NH}), 2205(\mathrm{CN}), 1664(\mathrm{CO}) \mathrm{cm}^{-1}$. MS: $\mathrm{m} / \mathrm{z}(\%)=573\left[\mathrm{M}^{+}\right]$. Anal. for $\mathrm{C}_{32} \mathrm{H}_{20} \mathrm{ClN}_{5} \mathrm{O}_{2} \mathrm{~S}$ : calcd. C, 66.95; H, 3.51; Cl, 6.18; N, 12.20; S, 5.59. found $\mathrm{C}, 66.47 ; \mathrm{H}, 3.28 ; \mathrm{Cl}, 5.92 ; \mathrm{N}, 11.78 ; \mathrm{S}, 5.24$.

\section{References}

[1] W. J. Houlihan, W. A. Remers and R. K. Brown, Wiley, New York, 1992.

[2] R. J. Sundberg, "The Chemistry of Indoles: Part I," Academic Press, New York, 1996.

[3] A. Casapullo, G. Bifulco, I. Bruno and R. Riccio, "New Bisindole Alkaloids of the Topsentin and Hamacanthin 
Classes from the Mediterranean Marine Sponge Rhaphisia Lacazei," Journal of Natural Products, Vol. 63, No. 4, 2000, pp. 447-451. doi:10.1021/np9903292

[4] B. Bao, Q. Sun, X. Yao, J. Hong, C. O. Lee, C. J. Sim, K. S. Im and J. H. Jung, "Bisindole Alkaloids from a Marine Sponge Spongosorites sp," Journal of Natural Products, Vol. 68, No. 5, 2005, pp. 711-715. doi:10.1021/np049577a

[5] T. Kouko, K. Matsumura and T. Kawasaki, "Total Synthesis of Marine Bisindole Alkaloids, (+)-Hamacanthins A, B and (-)-Antipode of Cis-Dihydrohamacanthin B," Tetrahedron, Vol. 61, 2005, pp. 2309-2318.

[6] K. Kaniwa, M. A. Arai, X. Li and M. Ishibashi, "Synthesis, Determination of Stereochemistry, and Evaluation of New Bisindole Alkaloids from the Myxomycete Arcyria ferruginea: An Approach for Wnt Signal Inhibitor," Bioorganic \& Medicinal Chemistry Letters, Vol. 17, No. 5, 2007, pp. 4254-4257. doi:10.1016/j.bmcl.2007.05.033

[7] L. H. Franco, E. B. K. Joffe, L. Puricelli, M. Tatian, A. M. Seldes and J. A. Palermo, "Indole Alkaloids from the Tunicate Aplidium Meridianum," Journal of Natural Products, Vol. 61, No. 9, 1998, pp. 1130-1132. doi:10.1021/np970493u

[8] A. A. R. Mohamed and E.-S. Mahmound, "Synthesis and Antitumor Activity of Indolylpyrimidines: Marine Natural Product Meridianin D Analogue," Bioorganic \& Medicinal Chemistry Letters, Vol. 15, No. 3, 2007, pp. 12061217. doi:10.1016/i.bmc.2006.11.023

[9] B. Jiang, C. G. Yang, W. N. Xiong and J. Wang, "Synthesis and Cytotoxicity Evaluation of Novel Indolylpyrimidines and Indolylpyrazines as Potential Antitumor Agents," Bioorganic \& Medicinal Chemistry Letters, Vol. 9, No. 5, 2001, pp. 1149-1154. doi:10.1016/S0968-0896(00)00337-0

[10] M. Gompel, M. Leost, E. B. K. Joffe, L. Puricelli, L. H. Franco, J. Palermo and L. Meijer, "Meridianins, a New Family of Protein Kinase Inhibitors Isolated from the Ascidian Aplidium meridianum," Bioorganic \& Medicinal Chemistry Letters, Vol. 14, No. 7, 2004, pp. 1703-1707. doi:10.1016/j.bmcl.2004.01.050

[11] I. Kawasaki, M. Yamashita and S. Ohta, "Successive Diarylation at the Carbon Positions of $1 H$-Imidazole and Its Application to the Total Synthesis of Nortopsentin D," Journal of the Chemical Society, Chemical Communications, No. 18, 1994, pp. 2085-2086. doi:10.1039/c39940002085

[12] I. Kawasaki, M. Yamashita and S. Ohta, "Total Synthesis of Nortopsentins A-D, Marine Alkaloids," Chemical and Pharmaceutical Bulletin, Vol. 44, No. 10, 1996, pp. 18311839.

[13] I. Kawasaki, H. Katsuma, Y. Nakayama, M. Yamashita and S. Ohta, "Total Synthesis of Topsentin, Antiviral and Antitumor Bis(indolyl)imidazole," Heterocycles, Vol. 48, 1998, pp. 1887-1901.

[14] H. H. Sun and J. Sakemi, "A Brominated (Aminoimidazolinyl)indole from the Sponge Discodermia Polydiscus," The Journal of Organic Chemistry, Vol. 56, No. 13, 1991, pp. 4307-4308. doi:10.1021/jo00013a045

[15] H. C. Vervoort, S. E. Richards-Gross, W. Fenical, A. Y. Lee and J. Clardy, "Didemnimides A-D: Novel, Predator-Deterrent Alkaloids from the Caribbean Mangrove Ascidian Didemnum conchyliatum," The Journal of Organic Chemistry, Vol. 62, No. 5, 1997, pp. 1486-1490. doi:10.1021/jo961789s

[16] S. Takahashi, T. Matsunaga, C. Hasegawa, H. Saito, D. Fujita, F. Kiuchi and Y. Tsuda, "Martefragin A, a Novel Indole Alkaloid Isolated from Red Alga, Inhibits Lipid Peroxidation," Chemical \& Pharmaceutical Bulletin, Vol. 46, No. 10, 1996, pp. 1527-1529.

[17] I. N'Diaye, G. Guella, G. Chiasera, Y. Mancini and F. Pietra, "Almazole A and Almazole B, Unusual Marine Alkaloids of an Unidentified Red Seaweed of the Family Delesseriaceae from the Coasts of Senegal," Tetrahedron Letters, Vol. 35, No. 27, 1994, pp. 4827-4830. doi:10.1016/S0040-4039(00)76979-6

[18] T. Bergmann, D. Schories and B. Steffan, "Alboinon, an Oxadiazinone Alkaloid from the Ascidian Dendrodoa grossularia," Tetrahedron, Vol. 53, 1997, pp. 2055-2060.

[19] S. Kohmoto, Y. Kashman, O. J. McConnell, K. L. Rinehart Jr., A. Wright and F. Koehn, "Dragmacidin, a New Cytotoxic Bis(Indole) Alkaloid from a Deep Water Marine Sponge, Dragmacidon sp," The Journal of Organic Chemistry, Vol. 53, No. 13, 1988, pp. 3116-3118. doi: $10.1021 /$ jo00248a040

[20] H. M. E. Hassaneen, H. M. Hassaneen and M. H. Elnagdi, "Enamines in Heterocyclic Synthesis: A Route to 4-Substituted Pyrazoles and Condensed Pyrazoles," Z. Naturforch., Vol. 59b, 2004, p. 1132.

[21] N. M. Elwan, H. M. E. Hassaneen and H. M. Hasssaneen, "Synthesis and Reactions of Indane-1,3-Dione-2-Thiocarboxanilides with Hydrazonoyl Halides and Active Chloromethylene Compounds," Heteroatom Chemistry, Vol. 13, No. 7, 2002, pp. 585-591. doi:10.1002/hc.10132

[22] M. A. A. Radwan, E. A. Ragab, N. M. Sabry and S. M. El-Shenawy, "Synthesis and Biological Evaluation of New 3-Substituted Indole Derivatives as Potential Anti-Inflammatory and Analgesic Agents," Bioorganic \& Medicinal Chemistry, Vol. 15, No. 11, 2007, pp. 3832-3841. doi:10.1016/j.bmc.2007.03.024

[23] H. M. E. Hassaneen and R. M. Pagni, "Synthesis of New 3-Substituted Indole Derivatives," Verlag der Zeitschrift Naturforschung, Vol. 65b, 2010, pp. 1491-1497.

[24] J. Slatt, I. Romero and J. Bergman, "Cyanoacetylation of Indoles, Pyrroles and Aromatic Amines with the Combination Cyanoacetic Acid and Acetic Anhydride," Synthesis, 2004, pp. 2760-2765.

[25] O. M. E. El-Dusouqui, M. M. Abdelkhalik, N. A. AlAwadi, H. Dib, B. J. George and M. H. Elnagdi, "Chemistry of 2-Arylhydrazonals: Utility of Substituted 2-Arylhydrazono-3-oxoalkanals as Precursors for 3-oxoalkanonitriles, 3-Aminoisoxazole and 1,2,3- and 1,2,4-Triazoles," Journal of Chemical Research, Vol. 2006, No. 5, 2006, pp. 295-302.

[26] I. M. Kenawi and M. H. Elnagdi, "DFT and FT-IR 
Analyses of Hydrogen Bonding in 3-Substistuted-3-oxoarylhydrazonopropanenitriles," Spectrochimica Acta Part A: Molecular and Biomolecular Spectroscopy, Vol. 65, No. 3-4, 2006, pp. 805-810.

doi:10.1016/j.saa.2006.01.017

[27] S. K. Mallick and A. R. Martin, "Synthesis and Antim- icrobial Evaluation of Some 5-(5-Nitrofurylidene)rhodanines, 5-(5-Nitrofurylidene)thiazolidine-2,4-diones, and Their Vinylogs," Journal of Medicinal Chemistry, Vol. 14, No. 6, 1971, pp. 528-532. doi:10.1021/jm00288a017

[28] S. R. Singh, Journal of the Indian Chemical Society, Vol. 52, 1975, pp. 734-735. 